Article

\title{
On the Capital Allocation Problem for a New Coherent Risk Measure in Collective Risk Theory
}

\author{
Hirbod Assa ${ }^{1}$, Manuel Morales ${ }^{2, *}$ and Hassan Omidi Firouzi ${ }^{3}$ \\ 1 Institute for Financial and Actuarial Mathematics, University of Liverpool, Liverpool L69 7ZX, UK; \\ assa@liverpool.ac.uk \\ 2 Department of Mathematics and Statistics, University of Montreal, CP. 6128 Succ. Centre-Ville, Montreal, \\ QC H3C 3J7, Canada \\ 3 Senior Enterprise Model Risk Analyst, Royal Bank of Canada, 200 Bay St, Toronto, ON M5J 2J1, Canada; \\ hassan.firouzi@rbccm.com \\ * Correspondence: morales@dms.umontreal.ca; Tel.: +1-514-343-6697
}

Academic Editor: José Garrido

Received: 25 May 2015; Accepted: 15 July 2016; Published: 16 August 2016

\begin{abstract}
In this paper we introduce a new coherent cumulative risk measure on a subclass in the space of càdlàg processes. This new coherent risk measure turns out to be tractable enough within a class of models where the aggregate claims is driven by a spectrally positive Lévy process. We focus our motivation and discussion on the problem of capital allocation. Indeed, this risk measure is well-suited to address the problem of capital allocation in an insurance context. We show that the capital allocation problem for this risk measure has a unique solution determined by the Euler allocation method. Some examples and connections with existing results as well as practical implications are also discussed.
\end{abstract}

Keywords: capital allocation; Euler allocation method; coherent risk measures; Lévy insurance processes; risk measures on the space of stochastic processes

\section{Introduction}

Collective risk theory has built upon the pioneering work of Filip Lundberg [1] and it concerns itself with the study the riskiness of an insurer's reserve as measured by the ruin probability and related quantities [2]. A large amount of literature now exists on such insolvency measures for a wide variety of models, the latest being the so-called Lévy insurance risk models [3,4].

Traditionally, collective risk theory focuses on the insurer's ability to manage the solvency of its reserve through the control of initial investment $x$. The mathematical tool often cited for such task is the probability of ruin. It is indeed a measure of the likelihood that an insurer's reserve would eventually be insufficient to cover its liabilities in the long run.

More precisely, consider the following general model for the risk reserve of an insurance company,

$$
R(t)=x+c t-X(t), \quad t \geq 0,
$$

where the aggregate claims process $X$ is a spectrally positive Lévy process with zero drift, with $X(0)=0$ and jump measure denoted by $v$. Moreover, $x$ is the initial reserve level and $c$ is a constant premium rate defined as

$$
c=(1+\theta) \mathbb{E}[X(1)]
$$

where $\theta>0$ is the security loading factor.

Then the associated ruin time is

$$
\tau_{x}:=\inf \{t \geq 0 \mid X(t)-c t \geq x\},
$$


and the infinite-horizon ruin probability can be defined by

$$
\psi(x):=\mathbb{P}^{x}\left(\tau_{x}<\infty\right),
$$

where $\mathbb{P}^{x}$ is short-hand notation for $\mathbb{P}(\cdot \mid X(0)=x)$.

Much of the literature in collective risk theory studies the problem of deriving expressions and reasonable approximations for the probability of ruin as a function of the initial reserve level $x$. This problem is addressed within an ever-growing set of models for the aggregate claims process. See [2] for a thorough account on the so-called ruin theory.

Within this framework, we engage our discussion from a risk management stand-point and with a capital allocation application in mind. Naturally, the ruin probability $\psi$ quantifies the solvency of the net-loss process $Y_{t}:=X_{t}-c t$ as a function of the initial reserve level $x$. In fact, we can define a risk measure $\rho_{\beta}: \mathcal{X} \longrightarrow[0,1]$ on a suitable model space $\mathcal{X}$ (say the space of bounded càdlàg stochastic processes $\mathcal{R}^{\infty}$ ). Let $Y_{t}=c t-X_{t}$ be the net-loss process associated with the reserve process (1), then

$$
\rho_{\beta}(Y) \longmapsto a:=\inf \{x \geq 0 \mid \psi(x) \leq \beta\}
$$

where $\psi$ is the associated ruin probability (4) and $\beta \in[0,1]$ represents a given tolerance to ruin.

One can interpret $a$ as the smallest initial level for which the process $R$ has an acceptable risk level, i.e., its associated ruin probability is less or equal to a tolerable figure $\beta$. Such risk measures have been recently studied (see [5]) and although they exhibit interesting properties, they lack the tractability of an efficient risk management tool. In fact, any meaningful risk management application, such as capital allocation, would be hard to implement using (5). Recently, other risk measures have been studied such as one based on the concept of area in red, which is a measure of how large the overall deficit of the company can be (see [6] for details). These new notions turn out to exhibit some interesting properties, yet the issue with these risk measures remains, they are very difficult to implement in a risk management problem such as capital allocation.

In this paper, we recover this idea of measuring the risk of an insurance risk process but with a view towards an application in the capital allocation problem. In fact, we reverse-engineer a risk measure with the sole purpose of addressing the non-trivial problem of capital allocation in a collective risk theory context. That is, we look at the aggregate loss from a number of, potentially dependent, lines of business and we give a way to allocate a portion of the overall risk to each component. More precisely, we define a coherent risk measure on a suitable subspace of the space of càdlàg processes, $R_{L}^{p}$, as a mapping $\rho: R_{L}^{p} \longrightarrow \mathbb{R}_{+}$(the precise description of the subspace in question, $R_{L^{\prime}}^{p}$, is given in Definition 1). Unlike (5), this measure is tractable enough and allows for a solution of the capital allocation problem within a suitable space of stochastic processes. This is carried out within the framework given by the theory of coherent and convex risk measures for stochastic processes. As it turns out, the problem of capital allocation for risk measures on the space of stochastic processes is a non-trivial one. In this paper we devise an ad-hoc risk measure for which the problem can be addressed. We ultimately give a definition of what we mean by capital allocation in this context and give an explicit solution for it. We also discuss connections between our approach and existing alternative solutions in the literature such as [7].

The outline of the paper is as follows. In Section 2, we give a brief literature review on the problem of capital allocation and risk measures for stochastic processes, thus giving a context to our contribution. In Section 3, we introduce the notion of Cumulative Entropic Value at Risk $\left(\mathrm{CEVaR}_{\beta}\right)$ as a coherent risk measure on the space of bounded stochastic processes and we explore some of its relevant features. In Section 4, we explore the capital allocation problem and give a theorem which characterizes the capital allocation set for these measures. In fact, we show that for the $\mathrm{CEVaR}_{\beta}$ risk measure the Euler allocation method is the only way to allocate the risk capital. We also discuss the connection between our approach and existing results for capital allocation in similar framework, namely that of [7]. In Section 5, we apply our results to study the capital allocation problem for a 
particular type of insurance Lévy risk process. Finally, in Section 6, we specialized our results to particular examples of Lévy insurance risk processes and give expressions for $\mathrm{CEVaR}_{\beta}$ in such cases.

\section{Literature Review}

We set to study risk measures for stochastic processes in the context of capital allocation applications. Our starting point is to build upon the notion of ruin measures in collective risk theory to design a more suitable measure of risk. There is indeed a fair amount of research on the question of how to define risk measures for stochastic processes. Among previous works on these issues we find, for instance, [8] and [9] where the authors work out risk measures on the space of random processes modeling the evolution of a certain financial position or [10] where they develop risk measures in a dynamic fashion. Indeed there is now a comprehensive theory of risk measures on the space of stochastic processes that draws from convex analysis, probability and the general theory of stochastic processes in order to build a mathematical framework for the quantification of economic risks in a dynamic fashion.

On the other hand, applications of risk measures defined on the space of stochastic processes are less abundant. In particular, the problem of capital allocation in such a framework is far from trivial. On the space of random variables, this problem is well-studied and it has been addressed in different ways. For an overview of several methods to tackle the problem of capital allocation in the insurance framework we refer to [11] or [12]. Moreover, in [13], a comparison of different combinations of risk measures and allocation methods can be found. For more recent references providing a good literature review on the problem, we refer to $[7,14]$.

The study of capital allocation problem can be traced back to the work of LeMaire [15] where the author studies the capital allocation problem in the game theory framework. Later on, in [16] we find that the author also uses a cooperative game theory approach to study the fair allocation principle for coherent risk measures. In [17], the author also studies the problem of capital allocation for random variables in a general framework. In [16,17], the authors propose the Euler principle for allocating the required capital under some technical assumptions. In [18], the author considers the particular case of multivariate normally distributed random vectors for risks and Tail Value at Risk (TVaR) as the risk measure. The author provides an explicit solution for the marginal based capital allocation. The authors in [19] extend the work done in [18] to the class of multivariate elliptical distributions. The author in [20] studies the problem for distortion risk measures. The results provided in [19] were also derived in [21] and the problem of capital allocation were studied for risks obtained by sums of normal as well as log-normal risk variables. In [22], the authors also study the capital allocation problem using default option with limited liability. Studying the problem for the class of convex risk measures was carried out in [23]. The authors in [24] study the capital allocation problem by introducing the class of weighted risk capital allocations. A different approach to the problem of capital allocation were introduced in [25] where the author introduces an axiomatic allocation framework to study the problem. In [26] the author studies the optimal capital allocation in a way to minimize the asymptotic ruin probability. A unifying framework for the capital allocation problem is developed in [7] where the authors use an approach relying "on an optimization argument, requiring that the weighted sum of measures for the deviations of the business unit's losses from their respective allocated capitals be minimized". Another study on this problem can be found in [27], where they propose a sensitivity analysis framework for internal risk models for the class of distortion risk measures. As a special case they study the problem of capital allocation for this class of risk measures under their sensitivity framework. For the most recent articles on the capital allocation problem we also refer to [28,29].

Unfortunately, when it comes to the space of stochastic processes, it is a different story. The problem of capital allocation for coherent risk measures on the space of stochastic processes turns out to be a more difficult task. A comprehensive understanding of the problem of capital allocation for coherent risk measures in this setting requires advanced notions and techniques from 
functional analysis as well as convex analysis. In fact, a formal treatment of the problem of capital allocation for coherent risk measures requires studying the weak sub-gradient set associated to the risk measure (see [30]). As it turns out, in order to get a good understanding of the sub-gradient set and its properties, we need a robust representation of the underlying risk measure which, in turn, requires studying of the dual space of $R_{L}^{p}$. Now, the fact that, we have a sophisticated topological structure to deal with for the dual space of $R_{L}^{p}$, makes it difficult to characterize the sub-gradient set and to give a solution to the problem of capital allocation. To the best of our knowledge, this problem has not been thoroughly studied for risk measures defined on the space of stochastic processes. We can only cite, [30], where the author discusses the problem of capital allocation for risk measures defined on the space of cádlág processes. Or, [6] where the authors study the capital allocation problem for a new risk measure that, as it turned out, it does not satisfy an axiomatic definition of coherent risk measures defined on stochastic processes proposed in [8]. As a drawback, the resulting solution of the capital allocation problem, does not follow an axiomatic definition of capital allocation. Moreover, neither the proposed risk measure, nor the capital allocation solution, have an explicit formula.

In this paper, we circumvent all these issues by giving an ad-hoc smooth explicit representation of a class of risk measures. Such construction leads naturally to an explicit solution for the capital allocation problem without having to make use of the advanced machinery from functional analysis. Based on [31] and [32], we design a risk measure on the space of bounded càdlàg processes that can capture the risk associated with the path-properties of an insurance model. We do this by extending the notion of Entropic Value at Risk, first introduced in [31], to a suitable space of stochastic processes. This measure allows us to explore the capital allocation problem in an insurance context. We show that the Euler allocation method is the only method to allocate the requiring capital for this risk measure. Moreover, we discuss a connection with existing results mainly with the results in [7].

\section{Cumulative Entropic Risk Measures}

Let $(\Omega, \mathcal{F}, \mathbb{P}, \overline{\mathcal{F}})$ be a filtered probability space. We consider the space $\mathcal{R}^{p}$ of stochastic processes on $[0, T]$ that are càdlàg, adapted and such that $X^{*}:=\sup _{[0, T]}\left|X_{t}\right| \in L^{p}(\Omega, \mathcal{F}, \mathbb{P})$, with $1 \leq p \leq \infty$. Furthermore, assume that $L^{1}(\Omega, \mathcal{F}, \mathbb{P})$ has a countable dense subset. Notice that, for any $1 \leq p \leq \infty$, the space $\mathcal{R}^{p}$ endowed with the norm $\|X\|_{\mathcal{R}^{p}}=\left\|X^{*}\right\|_{L^{p}}$, is a Banach space.

In [8] and [9] the authors developed the theory of convex risk measures on the space of $\mathcal{R}^{p}$ $\left(\rho: \mathcal{R}^{p} \longrightarrow \mathbb{R}_{+}\right)$. It is within this framework that we develop our approach. We start by defining a subset of $\mathcal{R}^{p}$ that serves our purposes and with which we will work for the rest of the paper.

Definition 1. We define the subspace $\mathcal{R}_{L}^{p}$ containing the processes $X \in \mathcal{R}^{p}$ with the following property.

$$
m_{t}(s)=\mathbb{E}\left[\exp \left(-s X_{t}\right)\right]<\infty, \quad s \geq 0,
$$

for $t \in[0, T]$.

The idea we put forward in this paper is to use a cumulative risk measure based on the Entropic Value at Risk that was defined in [31]. That is, following [30], we measure the risk of a random process $X \in \mathcal{R}_{L}^{p}$ by defining a cumulative risk measure $\rho: \mathcal{R}_{L}^{p} \longrightarrow \mathbb{R}_{+}$as follows. Let $\rho_{0}$ be a given risk measure on $L^{p}(\Omega, \mathcal{F}, \mathbb{P})$, i.e., $\rho_{0}: L^{p}(\Omega, \mathcal{F}, \mathbb{P}) \longrightarrow \mathbb{R}$, and let $\omega:[0, T] \longrightarrow \mathbb{R}^{+}$be a suitable weight function, i.e., $\int_{0}^{T} \omega(t) d t=1$. Then we can define a cumulative risk measure $\rho: \mathcal{R}_{L}^{p} \longrightarrow \mathbb{R}_{+}$based on $\rho_{0}$ as the weighted aggregate risk of a random process $X \in \mathcal{R}_{L}^{p}$. More precisely,

$$
\rho(X):=\int_{0}^{T} \rho_{0}\left(X_{t}\right) \omega(t) d t
$$


An interesting and meaningful choice for a weight function mentioned above can be a density function for a random time which captures important moments associated to the underlined process $X=\left(X_{t}\right)_{t \geq 0}$. Such constructions were proposed and studied in [30]. The features of such measures inherently depend on the choice of base risk measure $\rho_{0}$. In fact, if the risk measure $\rho_{0}$ is coherent then $\rho$ in (6) is coherent as well.

Theorem 1. Let $L^{p}(\Omega, \mathcal{F}, \mathbb{P})$ be the space of financial positions with finite $\left\|_{1}\right\|_{p}$ and $\rho_{0}$ be a coherent risk measure on $L^{p}(\Omega, \mathcal{F}, \mathbb{P})$. Then the risk measure $\rho: \mathcal{R}_{L}^{p} \longrightarrow \mathbb{R}_{+}$, given in $(6)$, is a coherent risk measure on the space $\mathcal{R}_{L}^{p}$.

Proof. First we show the positive homogeneity and translation invariance properties of $\rho$. For $\lambda>0$ and $m \in \mathbb{R}$ we have,

$$
\rho(\lambda X+m)=\int_{0}^{T} \rho_{0}\left(\lambda X_{t}+m\right) \omega(t) d t=\lambda \rho(X)-m \int_{0}^{T} \omega(t) d t
$$

which shows the positive homogeneity and translation invariance properties since $\int_{0}^{T} \omega(t) d t=1$.

As for monotonicity, if $X_{t} \leq Y_{t}$ a.s., then $\rho_{0}\left(X_{t}\right) \geq \rho_{0}\left(Y_{t}\right)$ for $t \in[0, T]$. Now, since $\omega$ is a positive real valued function, we have $\rho_{0}\left(X_{t}\right) \omega(t) \geq \rho_{0}\left(Y_{t}\right) \omega(t)$ for any $t \in[0, T]$ as well. This implies that $\rho(X) \geq \rho(Y)$ which proves the monotonicity.

Now using the subadditivity property of $\rho_{0}$ and since $\omega$ is a positive function we have,

$$
\rho_{0}\left(X_{t}+Y_{t}\right) \omega(t) \leq \rho_{0}\left(X_{t}\right) \omega(t)+\rho_{0}\left(Y_{t}\right) \omega(t),
$$

for $t \in[0, T]$. This directly implies the subadditivity property of $\rho$. i.e.,

$$
\rho(X+Y) \leq \rho(X)+\rho(Y) .
$$

In this paper, we propose to use the Entropic Value at Risk measure $\left(\mathrm{EVaR}_{\beta}\right)$ as our measure $\rho_{0}$ in (6). This yields an interesting family of risk measures on the space of bounded stochastic processes. Following $[31,33]$ we now give a first definition.

Definition 2. Let $X$ be a random variable in $L^{\infty}(\Omega, \mathcal{F}, \mathbb{P})$ with

$$
\mathbb{E}[\exp (-s X)]<\infty, \quad s>0 .
$$

Then the Entropic Value at Risk, denoted by $E V a R_{\beta}$, is given by

$$
\operatorname{EVaR}_{\beta}(X):=\inf _{s>0} \frac{\ln \mathbb{E}[\exp (-s X)]-\ln \beta}{s},
$$

for risk level $\beta \in(0,1)$.

We can generalize this definition for random variables in $L^{p}(\Omega, \mathcal{F}, \mathbb{P})$ for $p \geq 1$, as long as they meet (7). The following key result for $\mathrm{EVaR}_{\beta}$ can be found in [31].

Theorem 2. The risk measure $E V a R_{\beta}$ from Definition 2 is a coherent risk measure. Moreover, for any $X \in L^{\infty}(\Omega, \mathcal{F}, \mathbb{P})$ having property $(7)$, its dual representation has the form

$$
E V a R_{\beta}(X)=\sup _{f \in \mathcal{D}} \mathbb{E}_{\mathbb{P}}(-f X),
$$


where $\mathcal{D}=\left\{f \in L_{+}^{1}(\Omega, \mathcal{F}, \mathbb{P}) \mid \mathbb{E}_{\mathbb{P}}[f \ln (f)] \leq-\ln \beta\right\}$ and

$$
L_{+}^{1}(\Omega, \mathcal{F}, \mathbb{P})=\left\{f \in L^{1}(\Omega, \mathcal{F}, \mathbb{P}) \mid \mathbb{E}_{\mathbb{P}}(f)=1\right\} .
$$

For the proof we refer to [31].

If we use the risk measure (8) in our general definition of a cumulative risk measure (6), we naturally obtain a risk measure on the space $\mathcal{R}_{L}^{p}$ that would inherit some of the key features of the original risk measure.

We now formally introduce the concept of Cumulative Entropic Value at Risk, denoted by CEVaR ${ }_{\beta}$, on the space $\mathcal{R}_{L}^{p}$.

Definition 3. Let $X$ be a stochastic process in $\mathcal{R}_{L}^{p}$ and let $E V a R_{\beta}$ be the risk measure in Definition 2. Then, for a given weight function $\omega:[0, T] \longrightarrow \mathbb{R}^{+}$(i.e., $\int_{0}^{T} \omega(t) d t=1$ ), the Cumulative Entropic Value at Risk, denoted by $C E V a R_{\beta}$, is defined by

$$
\operatorname{CEVaR}_{\beta}(X)=\int_{0}^{T} \operatorname{EVaR}_{\beta}\left(X_{t}\right) \omega(t) d t .
$$

The main advantage of using (8) as our based measure is that the resulting cumulative risk measure (11) is tractable enough for a wide family of collective risk models. This comes from the fact that the expectation appearing in (8) is merely the Laplace exponent of the random variable $X_{t}$ (for $t \geq 0$ ). In collective risk theory, many of the models used for insurance reserves have closed-form Laplace transforms, in particular the so-called Lévy insurance risk processes. If the aggregate claims process is driven by a spectrally negative Lévy processes then a cumulative entropic risk measure based on the $\mathrm{EVAR}_{\beta}$ is an natural choice to work with in risk management applications.

The risk measure in Definition 3 belongs to the general framework of axiomatic risk measures on the space of stochastic processes developed in [8]. We now study some of its properties.

Corollary 1. The risk measure $C E V a R_{\beta}$, given in Definition 3, is a coherent risk measure on the space $\mathcal{R}_{L}^{p}$.

Proof. Since $\mathrm{EVaR}_{\beta}$ is of the form (6) with a coherent base risk measure $\rho_{0}$, it follows that $\mathrm{EVaR}_{\beta}$ is a coherent risk measure as a special case of Theorem 1.

Now, one can notice that in Definition 3 the weight function $\omega$ plays an important role. Different choices of weight functions would result in different cumulative Entropic risk measures. One can naturally think of $\omega$ as a density function that distributes a probability mass over the interval $[0, T]$. Interesting choices would be to use the density function $f_{\tau}$ of a suitable stopping time $\tau \in[0, T]$, like the first passage time or ruin time. This would penalize certain regions of the interval $[0, T]$ according to whether a certain meaningful event is more or less likely to occur over these regions.

For tractability purposes, in this paper, we use a uniform weight function, i.e., we consider $\omega(t)=\frac{1}{T}$. In the remaining of the paper we will be working with the following subfamily of CEVaR,

$$
\operatorname{CEVaR}_{\beta}(X)=\frac{1}{T} \int_{0}^{T} \operatorname{EVaR}_{\beta}\left(X_{t}\right) d t .
$$

Now, the object of our interest in this paper is to apply the CEVaR in (12) within an insurance context. That is, we would be interested in applying CEVaR in order to measure the risk of the net-loss process $X_{t}-c t$ in (1), where the aggregate claims process $X_{t}$ is a spectrally positive Lévy processes (or equivalently, the surplus process is modeled by a spectrally negative Lévy process). The following proposition enables us to include a subclass of spectrally negative Lévy processes having m.g.f in the space $\mathcal{R}^{p}$ for some $p \geq 1$. This would enable us to use $C E V a R$ with this class of processes. 
Proposition 1. Let $X$ be a càdlàg Lévy process with $X_{0}=0$ and let $p$ be a real number in $[1, \infty)$. Then, the following are equivalent.

1. $X$ is $L^{p}$-integrable.

2. $X_{t}^{*}=\sup _{0 \leq s \leq t}\left|X_{s}\right|$ is $L^{p}$-integrable.

Proof. This is a special case of Theorem 25.18 in [34].

Now that we have established, through Proposition 1, that the class of $L^{1}$-integrable spectrally negative Lévy processes having m.g.f is in the space $\mathcal{R}^{1}$; we can use such processes as models for the reserves of an insurance company and apply CEVaR in order to quantify risks.

One interesting feature is that the Cumulative Entropic Risk Measure has the advantage of being tractable enough for a large family of processes which have moment generating function and that can be used as models for the net-loss process in (1). Indeed, if our model for the net-loss process is a Lévy insurance model for which the moment generating function is readily available, computation of CEVaR is a somewhat straight-forward exercise. For instance, assume the net-loss process in (1) takes the form $Y_{t}=\mu t+\sigma W_{t}+X_{t}$, where $W_{t}$ is a standard Brownian motion, $\mu<0$ is the drift parameter, $\sigma>0$ is the and scale parameter, and $X_{t}$ is a gamma process with parameters $a, b>0$. Since $Y_{t}$ is a Lévy process, the moment generating function of $Z_{t}=-Y_{t}$ is

$$
\mathbb{E}\left(e^{-s Z_{t}}\right)=\mathbb{E}\left(e^{s Y_{t}}\right)=e^{\mu t s+\frac{1}{2} \sigma^{2} s^{2} t-t a \ln (1-s / b)} .
$$

In this case, to obtain $\operatorname{EVaR}_{\beta}\left(Z_{t}\right)$ we need to find $s^{*}$ from the following equation.

$$
\frac{2 t a}{b^{2}}\left(b s-s^{2}\right)+t a \ln \left(1-\frac{s}{b}\right)=s^{2} \sigma^{2} t, \quad s \geq 0 .
$$

The above equation is obtained by applying the moment generating function of $Z_{t}$ in the definition $\mathrm{EVaR}_{\beta}$ and by straight-forward differentiation with respect to $s$. Although there is no closed-form expression for the solution of this equation, once $s^{*}$ is obtained numerically, we can calculate $\operatorname{EVaR}_{\beta}\left(Z_{t}\right)$ by direct substitution $s^{*}$ in $(8)$. $\mathrm{CEVaR}_{\beta}(Z)$ can be obtained by direct integration of $\operatorname{EVaR}_{\beta}\left(Z_{t}\right)$ over $[0, T]$.

This is one example that illustrates how EVar and CEVaR can be computed for processes having a closed form moment generating function. More details and examples are given in a later section where we set up an insurance model based on Lévy processes to illustrate an application of our results in a capital allocation context.

\section{Capital Allocation}

We now study the problem of capital allocation in an insurance context with the coherent risk measure CEVaR that we introduced in Section 3. A discussion of the problem of capital allocation for $C E V a R$, which is a risk measure defined on $\mathcal{R}_{L^{\prime}}^{p}$ must start with an analysis of such problem for EVaR, which is a risk measure on a subspace of $L^{\infty}(\Omega, \mathcal{F}, \mathbb{P})$.

Finding the capital allocation for a risk measure on the space of stochastic processes typically requires knowledge of its robust representation and its subgradient set (see [32] for a detailed account on this problem). This robust representation is typically a hard problem in the space $\mathcal{R}_{L}^{p}$ that normally requires advanced functional analysis tools. In the case of $E V a R$ we propose to tackle the problem of finding the capital allocation for CEVaR by finding first the capital allocation for EVaR and then use the linear relation between EVaR and CEVaR to get the capital allocation for CEVaR.

We first give some definitions that will be needed throughout this section. 
Definition 4. Let $\rho$ be a coherent risk measure defined on $L^{\infty}(\Omega, \mathcal{F}, \mathbb{P})$. Now let $\mathcal{D} \subset L_{+}^{1}$ be the largest set for which the following robust representation holds true for $\rho$.

$$
\rho(X)=\sup _{f \in \mathcal{D} \subset L_{+}^{1}} \mathbb{E}_{\mathbb{P}}(-f X) \quad \forall X \in L^{\infty}(\Omega, \mathcal{F}, \mathbb{P})
$$

where $L_{+}^{1}$ is the set defined in (10). The set $\mathcal{D}$ is called the determining set of $\rho$ (see [35]).

The following definition is taken from [36].

Definition 5. Let $\rho$ be a coherent risk measure defined on $L^{\infty}(\Omega, \mathcal{F}, \mathbb{P})$ with determining set $\mathcal{D} \subset L_{+}^{1}$. Let $X \in L^{\infty}(\Omega, \mathcal{F}, \mathbb{P})$. A function $f \in \mathcal{D}$ is called an extreme function for $X$ if $\rho(X)=\mathbb{E}_{\mathbb{P}}(f X) \in(-\infty, \infty)$. The set of extreme functions will be denoted by $\chi_{\mathcal{D}}(X)$.

The following result is taken from [36] and gives conditions for the set of extreme functions defined above to be non-empty.

Proposition 2. Let $\mathcal{D} \subset L_{+}^{1}$ be the determining set of a given coherent risk measure $\rho$ on $L^{\infty}(\Omega, \mathcal{F}, \mathbb{P})$. Now consider the following set.

$$
L^{1}(\mathcal{D}):=\left\{X \in L^{\infty}(\Omega, \mathcal{F}, \mathbb{P}) \mid \lim _{n \rightarrow \infty} \sup _{f \in \mathcal{D}} \mathbb{E}_{\mathbb{P}}\left[f|X| \mathbb{I}_{\{|X|>n\}}\right]=0\right\}
$$

If the determining set $\mathcal{D}$ is weakly compact and $X \in L^{1}(\mathcal{D})$, then the set of extreme functions for $X$ is not empty, i.e., $\chi_{\mathcal{D}}(X) \neq \varnothing$.

Now, we turn our attention to the concept of capital allocation. Consider a vector of risks $X=\left(X^{1}, \ldots, X^{d}\right)$, such that $X^{i} \in L^{\infty}(\Omega, \mathcal{F}, \mathbb{P})$ for $i=1, \ldots, d$, are random variables representing the cash flow or risk exposure of a portfolio consisting of $d$ risky positions or departments.

Given a coherent risk measure $\rho$ on $L^{\infty}(\Omega, \mathcal{F}, \mathbb{P})$, we now look at the problem of how to allocate the total risk of the portfolio $\rho\left(X^{1}+\cdots+X^{d}\right)$ among the different departments such that the individual risk of each one of them is properly measured.

The following formal definition of capital allocation was proposed by [37] and [38] and it is the one we set out to study in this paper. In fact, the following gives a mathematical definition of capital allocation for a coherent risk measure.

Definition 6. Consider a coherent risk measure $\rho$ on $L^{\infty}(\Omega, \mathcal{F}, \mathbb{P})$ and a vector of risks $X=\left(X^{1}, \ldots, X^{d}\right)$ such that $X^{i} \in L^{\infty}(\Omega, \mathcal{F}, \mathbb{P})$ for $i=1, \ldots, d$. A fair capital allocation for $X$ is a vector $\left(K^{1}, \ldots, K^{d}\right) \in \mathbb{R}^{d}$ such that

1.

2.

$$
\sum_{i=1}^{d} K^{i}=\rho\left(\sum_{i=1}^{d} X^{i}\right)
$$

$$
\sum_{i=1}^{d} h^{i} K^{i} \leq \rho\left(\sum_{i=1}^{d} h^{i} X^{i}\right), \quad \forall h=\left(h^{1}, \ldots, h^{d}\right) \in \mathbb{R}_{+}^{d} .
$$

The first condition is called the full allocation property and it simply states the fact that the total risk of the whole portfolio should be the aggregated risks of each department. The second condition is called the linear diversification property of capital allocation. In fact, this condition has a one to one correspondence with the positive homogeneity and subadditivity of a coherent risk measure $\rho$ (see [25]). Since we work in this paper with a coherent risk measure it is somehow natural to adopt this definition of capital allocation. 
The following is an interesting result characterizing the set of possible such capital allocations and it is adapted from [36].

Theorem 3. Let $\mathcal{D} \subset L_{+}^{1}$ be the determining set of a given coherent risk measure $\rho$ on $L^{\infty}(\Omega, \mathcal{F}, \mathbb{P})$ and let $X=\left(X^{1}, \ldots, X^{d}\right)$ be a vector such that $X^{i} \in L^{\infty}(\Omega, \mathcal{F}, \mathbb{P})$ for $i=1, \ldots, d$. Consider the following set

$$
G=\overline{\left\{\left(\mathbb{E}_{\mathbb{P}}\left(-f X^{1}\right), \ldots, \mathbb{E}_{\mathbb{P}}\left(-f X^{d}\right)\right) \mid f \in \mathcal{D}\right\}} \subset \mathbb{R}^{d} .
$$

The set $U \subset \mathbb{R}^{d}$ of capital allocations for $X=\left(X^{1}, \ldots, X^{d}\right)$, satisfying Definition 6 , is convex and bounded and it has the form

$$
U=\underset{x \in G}{\operatorname{argmax}}<e, x>
$$

where $<\cdot, \cdot>$ is the inner product in $\mathbb{R}^{d}, e=(1, \ldots, 1)$ and argmax is the set of points of $G$ for which $<e, x>$ attains its maximum value.

If moreover, $X^{1}, \ldots, X^{d} \in L^{1}(\mathcal{D})$ and $\mathcal{D}$ is weakly compact, then $U$ can be identified to be

$$
U=\left\{\left(\mathbb{E}_{\mathbb{P}}\left(-f X^{1}\right), \ldots, \mathbb{E}_{\mathbb{P}}\left(-f X^{d}\right)\right) \mid f \in \chi_{\mathcal{D}}\left(\sum_{i=1}^{d} X^{i}\right)\right\} .
$$

Proof. In [36], the author provides a proof of the theorem for coherent utility functions. The result follows by noticing that, for a given coherent risk measure $\rho$, if we set $\rho^{*}(X):=-\rho(-X)$ we obtain a coherent utility function and the result in [36] holds. So, from $\rho(X)=-\rho^{*}(-X)$ the results for the statement of our theorem holds.

The set $G \subset \mathbb{R}^{d}$ in Theorem 3 is called the generator for $X$ and $\rho$ (see [36]). The following corollary gives a condition on $G$ for the uniqueness of the capital allocation.

Corollary 2. Under the conditions of Theorem 3. If moreover, $G \subset \mathbb{R}^{d}$ is strictly convex (i.e., its interior is non-empty and its border contains no interval), then there is a unique capital allocation satisfying Definition 6.

Proof. See [36] for a proof in terms of coherent utility functions.

\subsection{CEVaR and the Capital Allocation Problem}

Our main goal in this paper is to apply cumulative entropic risk measure in a capital allocation problem. So far, we have discussed key notions of the capital allocation problem for a risk measure on $L^{\infty}(\Omega, \mathcal{F}, \mathbb{P})$. In this subsection, we apply these results in order to give an answer to the problem of capital allocation for $C E V a R$ which is a risk measure on $\mathcal{R}_{L}^{p}$. Notice that this is a somewhat more complicated problem since there is a dynamic component to this problem. Here, this is overcome by the cumulative property of $\mathrm{CEVa} R_{\beta}$. We start by extending Definition 6 to the more general notion of capital allocation with respect to a coherent risk measure on the space $\mathcal{R}_{L}^{p}$. The following definition is taken from [39].

Definition 7. Let $\left(X_{t}^{1}, \ldots, X_{t}^{d}\right)_{t \in[0, T]}$ be d random processes in $\mathcal{R}_{L}^{p}$ representing d financial positions or departments. Moreover, consider a coherent risk measure $\rho: \mathcal{R}_{L}^{p} \longrightarrow \mathbb{R}_{+}$defined on the space $\mathcal{R}_{L}^{p}$. A fair capital allocation for $\left(X_{t}^{1}, \ldots, X_{t}^{d}\right)_{t \in[0, T]}$ with respect to $\rho$ is a vector $\left(L^{1}, \ldots, L^{d}\right) \in \mathbb{R}^{d}$ such that

1.

$$
\sum_{i=1}^{d} L^{i}=\rho\left(\sum_{i=1}^{d} X^{i}\right)
$$


2.

$$
\sum_{i=1}^{d} h^{i} L^{i} \leq \rho\left(\sum_{i=1}^{d} h^{i} X^{i}\right), \quad \forall h=\left(h^{1}, \ldots, h^{d}\right) \in \mathbb{R}_{+}^{d},
$$

where $\sum_{i=1}^{d} X^{i}$ denotes the process $\left(\sum_{i=1}^{d} X_{t}^{i}\right)_{t \in[0, T]}$.

In this section, we show how a capital allocation satisfying Definition 7 can be obtained when using CEVaR as risk measure. In fact we show that the border of the set $\mathcal{D}$ in the robust representation (13) for $\mathrm{EVa}_{\beta}$ is not a convex set so leads to the fact that the border of the set $G$ in (15) is not a convex set as well. This yields to the uniqueness of the capital allocation for EVaR (Corollary 2) as well as for CEVaR.

Theorem 4. Let $D$ be the determining set in the robust representation (9) for $E V a R_{\beta}$. Then the convex combination of elements of $\partial \mathcal{D}$ is never an element of $\partial \mathcal{D}$ where $\partial \mathcal{D}=\left\{f \in L_{+}^{\infty}: \mathbb{E}_{\mathbb{P}}(f \ln (f))=-\ln \beta\right\}$.

Proof. It is sufficient to show that for any $\lambda \in[0,1]$ and any two functions $f$ and $g$ in $\partial \mathcal{D}$, the function $\lambda f+(1-\lambda) g$ is not in $\partial \mathcal{D}$. Define the function $H$ on the space of positive real line taking real values as follows.

$$
H(x):=x \ln x,
$$

for all $x \in \mathbb{R}^{+}$.

It is clear that the function $H$ is strictly convex on the positive real line. Since, $H^{\prime}(x)=\ln x+1$ and $H^{\prime \prime}(x)=\frac{1}{x}>0$ for all $x \in \mathbb{R}_{+}$. Now again we define a new function $K$ on $[0,1]$ with its values in $\mathbb{R}$ by using the composition function $H(\lambda f+(1-\lambda) g)$ as follows.

$$
K(\lambda)=E_{\mathbb{P}}(H(\lambda f+(1-\lambda) g))
$$

for the fixed functions $f$ and $g$ in $\partial \mathcal{D}$. Notice that we use a slight abuse of notation, here $H(\lambda f+(1-\lambda) g)$ is to be understood point-wise. That is, for $x \in \mathbb{R}$, the function $H(\lambda f+(1-\lambda) g) \longrightarrow H(\lambda f(x)+(1-\lambda) g(x))$.

If we take the first and second derivatives for the function $K$, we see that this function is strictly convex too. $K^{\prime}(\lambda)=E_{\mathbb{P}}\left((f-g)\left(H^{\prime}(\lambda f+(1-\lambda) g)\right)\right.$ and $K^{\prime \prime}(\lambda)=E_{\mathbb{P}}\left((f-g)^{2}\left(H^{\prime \prime}(\lambda f+(1-\lambda) g)\right)=\right.$ $\frac{(f-g)^{2}}{\lambda f+(1-\lambda) g}>0$. Now, considering $K(0)=E_{\mathbb{P}}(H(f))$ and $K(1)=E_{\mathbb{P}}(H(g))$ along with the strictly convexity of the function $K$, we come up with the inequality

$$
K(\lambda)=E_{\mathbb{P}}(H(\lambda f+(1-\lambda) g))<-\ln \beta \quad \forall \lambda \in(0,1) .
$$

This proves our assertion.

Now, we are going to characterize the capital allocation satisfying Definition 7 with respect to $\mathrm{CEVa}_{\beta}$ given by (11). Notice that this seems to be a more complicated problem since CEVa $R_{\beta}$ is a risk measure defined on the space of stochastic processes $\mathcal{R}_{L}^{p}$. However, this is possible thanks to the cumulative property of $\mathrm{CEVa} R_{\beta}$. In fact, this enables us to study the problem of capital allocation for $\mathrm{CEVa} R_{\beta}$ through studying the same problem for $\mathrm{EVa}_{\beta}$. A characterization theorem for the problem of capital allocation for coherent risk measures on space of random variables has been proved by [16] in the context of game theory. But in the following theorem, for the sake of completeness, we provide a different proof for the capital allocation for $\mathrm{EVa}_{\beta}$ using different approach.

Theorem 5. Let $\left(X_{t}^{1}, \ldots, X_{t}^{d}\right)_{0 \leq t \leq T}$ be a vector such that each $\left(X_{t}^{i}\right)_{0 \leq t \leq T} \in \mathcal{R}_{L}^{p}$ (for $\left.i=1, \ldots, d\right)$ represents the cash-flow or risk exposure from one risk position or department at time $t \in[0, T]$. We denote by $X_{t}=\sum_{i=1}^{d} X_{t}^{i}$ the portfolio-wide cash-flow produced at time $t \in[0, T]$. Furthermore, define the function $f_{\rho}^{t}\left(u_{1}, \ldots, u_{d}\right)=\rho\left(\sum_{i=1}^{d} u_{i} X_{t}^{i}\right)$ where $\rho$ is $E V a R_{\beta}$ as defined in Definition 2. Then, the capital allocation 
satisfying Definition 7 over the period $[0, T]$, with respect to $C E V a R_{\beta}$ as defined in (11), is determined uniquely for $i=1, \ldots, d$ by

$$
L^{i}=\int_{0}^{T} K_{t}^{i} \omega(t) d t
$$

where $K_{t}^{i}$ is

$$
K_{t}^{i}=\left.\frac{d \rho}{d h}\left(X_{t}+h X_{t}^{i}\right)\right|_{h=0}=\frac{\partial}{\partial u_{i}} f_{\rho}^{t}(1, \ldots, 1) \quad 1 \leq i \leq d, \quad t \in[0, T] .
$$

Proof. From the definition of $C E V a R_{\beta}$ it is clear that

$$
\operatorname{CEVaR}_{\beta}\left(\left(\sum_{i=1}^{d} X_{t}^{i}\right)_{t \in[0, T]}\right)=\int_{0}^{T} \operatorname{EVaR}_{\beta}\left(\sum_{i=1}^{d} X_{t}^{i}\right) \omega(t) d t=\int_{0}^{T} \operatorname{EVaR}_{\beta}\left(X_{t}\right) \omega(t) d t .
$$

Because of the linear property of integral, studying the problem of capital allocation, both existence and uniqueness of capital allocation, for $C E V a R_{\beta}$ can be reduced to study this problem for $E V a R_{\beta}$. Now, we show that $K_{t}^{i}$ provided in the theorem is the capital allocation for $E V a R_{\beta}$ and the vector $\left(X_{t}^{1}, \ldots, X_{t}^{d}\right)$ for a fixed $t \in[0, T]$. For this first of all we show that the possible capital allocations for the vector $\left(X_{t}^{1}, \ldots, X_{t}^{d}\right)$ are those belonging to the following set.

$$
A_{t}:=\left\{x \in G \mid f_{\rho}^{t}(V)-f_{\rho}^{t}(e) \geq\langle x, V-e\rangle, \quad \forall V \in \mathbb{R}^{d}\right\},
$$

where $G$ is given by $(15), e=(1, \ldots, 1)$ and $<\ldots>$ is the inner product in $\mathbb{R}^{d}$. To show this, we assume that $x \in G$ is a capital allocation for the vector $\left(X_{t}^{1}, \ldots, X_{t}^{d}\right)$. Thus, we have $f_{\rho}^{t}(e)=\langle e, x\rangle$. Then, for every $V \in \mathbb{R}^{d}$ we have $f_{\rho}^{t}(V)-f_{\rho}^{t}(e) \geq\langle x, V-e\rangle$. Therefore, $x \in A_{t}$. Now, assume that $x \in A_{t}$, then $f_{\rho}^{t}(V)-f_{\rho}^{t}(e) \geq\langle x, V-e\rangle$ for all $V \in \mathbb{R}^{d}$. By replacing $V=2 e$ and $V=\frac{1}{2} e$ into the last inequality we get $f_{\rho}^{t}(e)=\langle e, x\rangle$. Therefore, the set of capital allocation associated to the vector $\left(X_{t}^{1}, \ldots, X_{t}^{d}\right)$ is $A_{t}$. Since, the risk measure $\operatorname{EVaR}_{\beta}$ is positive homogeneous, i.e., for all $\lambda>0$ we have $\operatorname{EVaR}_{\beta}\left(\lambda X_{t}\right)=\lambda \operatorname{EVaR}_{\beta}\left(X_{t}\right)$, we deduce that the function $f_{\rho}$ above is a homogeneous function. So, by Euler's theorem on homogeneous functions we have $f_{\rho}^{t}\left(u_{1}, \ldots, u_{n}\right)=\sum_{i=1}^{d} u_{i} \frac{\partial}{\partial u_{i}} f_{\rho}^{t}\left(u_{1}, \ldots, u_{d}\right)$ which implies

$$
f_{\rho}^{t}(1, \ldots, 1)=\sum_{i=1}^{d} \frac{\partial}{\partial u_{i}} f_{\rho}^{t}(1, \ldots, 1)
$$

for $\left(u_{1}, \ldots, u_{n}\right)=(1, \ldots, 1)$. Because $\operatorname{EVaR}_{\beta}\left(X_{t}\right)$ has smooth explicit representation so partial derivative $\frac{\partial}{\partial u_{i}} f_{\rho}^{t}(1, \ldots, 1)$ exists for all $i=1, \ldots, d$. Now, referring to [36] we can see that $x=\left(\frac{\partial}{\partial u_{i}} f_{\rho}^{t}(1, \ldots, 1)\right)_{1 \leq i \leq d}$ belongs in $G$ as well as in $A_{t}$. This shows that gradient of $f_{\rho}^{t}$ evaluated at $e=(1, \ldots, 1)$ is one choice for capital allocation of the vector $\left(X_{t}^{1}, \ldots, X_{t}^{d}\right)$. To show the uniqueness we refer to any classical convex analysis textbook to see when the gradient exists then the set $A_{t}$ is a singleton [40]. i.e., the gradient of $f_{\rho}^{t}$ evaluated at $e=(1, \ldots, 1)$ is the only possible allocation for the vector $\left(X_{t}^{1}, \ldots, X_{t}^{d}\right)$. Now to get the capital allocation for $C E V a R_{\beta}$ we just need to multiply $K_{t}^{i}$ by $\omega(t)$ and take integral w.r.t $t$. This finishes the proof.

Corollary 3. Under the assumptions of Theorem 5, the capital allocated to the department $i^{\text {th }}$ for the cumulative risk measure $C E V a R_{\beta}$ given by (12) is

$$
L^{i}=\frac{1}{T} \int_{0}^{T} K_{t}^{i} d t
$$

where $K_{t}^{i}$ is given by Equation (19). 
Proof. By replacing $\omega(t)=\frac{1}{T}$ into Equation (18) we get the required capital for the department $i^{t h}$.

Theorem 5, gives us a solution to the problem of capital allocation for stochastic processes over a finite time period $[0, T]$. Interesting enough, unlike other solutions to this problem, this capital allocation can be readily computed for a large family of processes. But before we turn our attention to an application of our results, we elaborate further on the connection of our results and existing alternatives for capital allocation in similar contexts, namely the approach in [7].

\subsection{Connection to other Optimum Capital Allocation Principles}

Within our framework, Theorem 5 and Corollary 3 give a solution the capital allocation problem described in Definition 7. This can be seen as a way to circumvent technical difficulties associated with the allocation problem within the space of stochastic processes which are non-trivial. Now, we would like to briefly compare our approach to existing alternatives in the literature. Indeed, the problem of static capital allocation for random variables in a general optimization framework has been developed in [7]. The authors in [7] derive the optimal capital allocation for different business lines or sub portfolios via solving the following minimization problem.

$$
\min _{K_{1}, \ldots, K_{n}} \sum_{j=1}^{n} v_{j} \mathbb{E}_{P}\left[\zeta_{j} D\left(\frac{X_{j}-K_{j}}{v_{j}}\right)\right], \quad \text { such that } \sum_{j=1}^{n} K_{j}=K,
$$

where the $X_{j}$ are $\mathrm{n}$ individual losses, the $v_{j}$ are non-negative real numbers such that $\sum_{j=1}^{n} v_{j}=1$, the $\zeta_{j}$ are non-negative random variables such that $\mathbb{E}_{P}\left[\zeta_{j}\right]=1$, D is a non-negative function, and $K$ is the given total risk capital. Specializing $D(x)=x^{2}$, the authors reduce Equation (22) to the quadratic version of it and provide a closed form solution for $K_{j}$ as follows,

$$
K_{j}=\mathbb{E}_{P}\left(\zeta_{j} X_{j}\right)+v_{j}\left(K-\sum_{j=1}^{n} \mathbb{E}_{P}\left(\zeta_{j} X_{j}\right)\right), \quad 1 \leq j \leq n .
$$

It is worth pointing out that this proposed capital allocation solution in Equation (23) holds for the static framework, i.e., it provides the solutions for the problem of capital allocation for risk measures defined on the space of random variables. In the following we elaborate further on the connection between Equation (23) and the presented method for solving the capital allocation problem in this paper.

Setting $K=\operatorname{EVaR}_{\beta}\left(\sum_{j=1}^{n} X_{j}\right)$ and referring to Definition 6 and Corollary 2, it can be seen that $\zeta_{j}=f$ for all $1 \leq j \leq n$ where $f$ is the Radon-Nikodym derivative associated to the robust representation of $\operatorname{EVaR}_{\beta}\left(\sum_{j=1}^{n} X_{j}\right)$.

Now, if we compare Equation (23) with Equation (17) and we use Corollary 2, we can see that the capital allocation associated to each $X_{j}$ is uniquely determined by $K_{j}=\mathbb{E}_{P}\left(f X_{j}\right)$. One just has to remember that in Equation (17) we considered $X^{j}$ as profit and loss (P\&L) random variables which explains the "-" signs in Equation (17). Therefore, interestingly enough, Equation (23) is reduced to $K_{j}=\mathbb{E}_{P}\left(f X_{j}\right)$.

Now, in practice it is not an easy task to derive the function $f$ and, consequently, to obtain a closed form for $K_{j}$ in this setting. Other alternatives need to be adapted to find $K_{j}$ like Euler allocation method. Under this light, in Section 4.1 we have somewhat developed and extended the quadratic optimal capital allocation problem proposed in [7] for a class of risk measures defined on a subspace of stochastic processes, $\mathcal{R}_{L}^{p}$. Due to the closed form representation of the underlying class of risk measures, we derived solutions for the problem of quadratic capital allocation without restoring the complicated mathematical tools. 


\section{Capital Allocation for Insurance Lévy Risk Processes}

We now illustrate how Corollary 3 can be used to give an answer to the capital allocation problem for an insurance risk process. We consider here an insurance company consisting of $n$ departments. For each department, we let $R_{t}^{i}$ be a risk reserve process of the form (1). In other words, $R_{t}^{i}=x^{i}-Y_{t}^{i}$ where $Y_{t}^{i}=X_{t}^{i}-c^{i} t$ denotes the net-loss process related to the $i$-th department. We recall that $x^{i}$ is the initial reserve, $c^{i}$ is the loaded premium and $X_{t}^{i}$ is a model for the aggregate claims while the index $i$ refers to one of the $n$ departments. In order to allow for a more rich description of an insurance company, we can think of a similar setting to that used in the CreditRisk+ Model (see [41]) where the aggregate claims process $X_{t}^{i}$ is being described by $m$ independent systemic risk factors $W_{t}^{1}, \ldots, W_{t}^{m}$ which are themselves spectrally positive Lévy processes. Alternatively, one can think of the aggregate amount paid out by the department $i$ as being composed of fractions of $m$ independent classes of claims. That is, let $W_{t}^{1}, \ldots, W_{t}^{m}$ be $m$ independent spectrally positive Lévy process modeling aggregate claims of $m$ different types. In this perspective, one can think for instance of claims associated with car accidents, home damage, medical insurance, etc. From either perspective, the aggregate claims $X_{t}^{i}$ paid out by the $i$-th department would be a linear combination of some of these $W_{t}^{j}$ systemic claims processes. For example, consider aggregate claims produced by a car insurance contract. We suppose that one department will pay out property damage coverage (a fraction of the aggregate claims from the contract) while another department will pay out third-party liability costs (another fraction of the aggregate claims from the contract).

Mathematically, we let $W_{t}^{1}, \ldots, W_{t}^{m}$ be $m$ independent spectrally positive Lévy processes having moment generating function(m.g.f) for $j=1, \ldots, m$. Now, we let each $X_{t}^{i}$ to be a linear combination of some, or all, of the $W_{t}^{1}, \ldots, W_{t}^{m}$, i.e.,

$$
X=\left(\begin{array}{c}
X_{t}^{1} \\
X_{t}^{2} \\
\vdots \\
X_{t}^{n}
\end{array}\right)=\left(\begin{array}{ccc}
a_{11} & \ldots & a_{1 m} \\
a_{21} & \ldots & a_{2 m} \\
\vdots & \ldots & \vdots \\
a_{n 1} & \ldots & a_{n m}
\end{array}\right)\left(\begin{array}{c}
W_{t}^{1} \\
W_{t}^{2} \\
\vdots \\
W_{t}^{m}
\end{array}\right),
$$

where $a_{i j}$ 's are non-negative real numbers for $1 \leq i \leq n$ and $1 \leq j \leq m$.

We point out that we chose this structure because it admits a neat solution for the capital allocation problem through Theorem 5. One can always fall back on the more simple case where each department pays out one, and only one, type of claims as oppose to paying fractions of different types of claims. This would correspond to having $n=m$ and a diagonal matrix in (24) with all elements in the diagonal equal to one yielding $X_{t}^{i}=W_{t}^{i}$ for all $i$. We also point out that this construction endows the processes $R^{i}$ s with a dependence structure through the aggregate claims $X^{i}$ s. Now, the main objective would be to identify the capital allocation for each net-loss process $c^{i} t-X_{t}^{i}$ as measured by $\mathrm{CEVaR}_{\beta}$. The next result is one of the main contribution of our paper.

Theorem 6. Consider $n$ risk processes such that $\left(R_{t}^{i}\right)_{0 \leq t \leq T} \in \mathcal{R}_{L^{\prime}}^{p}$ for $i=1, \ldots, n$. Now, let such $R_{t}^{i}=x^{i}+c^{i} t-X_{t}^{i}$ where the aggregate risk processes $X_{t}^{i}$ be those defined in (24). Then the capital allocation that satisfies Definition 7 over the time period $[0, T]$, for each net-loss process $c^{i} t-X_{t}^{i}$ and with respect to the risk measure $C E V a R_{\beta}$ in (12) is

$$
L^{i}=\frac{1}{T} \int_{0}^{T} K_{t}^{i} d t-c^{i} \frac{T}{2}
$$

where

$$
K_{t}^{i}=t \sum_{j=1}^{m} a_{i j} \phi_{j}^{\prime}\left(s^{*} \sum_{k=1}^{n} a_{k j}\right), \quad t \in[0, T]
$$

and $\mathbb{E}\left(e^{s W_{1}^{j}}\right)=e^{\phi_{j}(s)}$ for $s \geq 0, \phi_{j}^{\prime}(0) \geq 0,1 \leq i \leq n$ and $1 \leq j \leq m$. 
Proof. First we want to find the capital allocation with respect to the risk measure $E V a R_{\beta}$ before applying Theorem 5. For any coherent risk measure $\rho$ defined on $L^{\infty}(\Omega, \mathcal{F})$, we have, by the cash-invariant property, that, for each $t \in[0, T]$

$$
\rho\left(\sum_{i=1}^{n} Y_{t}^{i}\right)=\rho\left(\sum_{i=1}^{n}-X_{t}^{i}\right)-\sum_{i=1}^{n} c^{i} t
$$

That is, in order to find the capital allocation (at $t \in[0, T]$ ) in this setting with respect to a coherent risk measure (in particular for $\mathrm{EVa}_{\beta}$ ), we just need to find the capital allocation for each claim process $X_{t}^{i}$.

For a given coherent risk measure $\rho$ on $L^{\infty}(\Omega, \mathcal{F})$, let us define the function $f_{\rho}^{t}\left(u_{1}, \ldots, u_{n}\right):=\rho\left(\sum_{i=1}^{n}-u_{i} X_{t}^{i}\right)$ for $t \in[0, T]$. Taking into account the structure of the processes $X_{t}^{1}, \ldots, X_{t}^{n}$, we can write, for $t \in[0, T]$

$$
\begin{aligned}
f_{\rho}^{t}\left(u_{1}, \ldots, u_{n}\right) & =\rho\left(\sum_{i=1}^{n}-u_{i} X_{t}^{i}\right) \\
& =\rho\left(-\left(\left(\sum_{j=1}^{m} u_{1} a_{1 j} W_{t}^{j}\right)+\left(\sum_{j=1}^{m} u_{2} a_{2 j} W_{t}^{j}\right)+\cdots+\left(\sum_{j=1}^{m} u_{n} a_{n j} W_{t}^{j}\right)\right)\right) \\
& =\rho\left(-\left(\left(\sum_{i=1}^{n} u_{i} a_{i 1}\right) W_{t}^{1}+\left(\sum_{i=1}^{n} u_{i} a_{i 2}\right) W_{t}^{2}+\cdots+\left(\sum_{i=1}^{n} u_{i} a_{i n}\right) W_{t}^{m}\right)\right) .
\end{aligned}
$$

If we let

$$
d_{j}=\sum_{k=1}^{n} u_{k} a_{k j}
$$

we can write a more compact form

$$
f_{\rho}^{t}\left(u_{1}, u_{2}, \ldots, u_{n}\right)=\rho\left(-\left(d_{1} W_{t}^{1}+d_{2} W_{t}^{2}+\cdots+d_{m} W_{t}^{m}\right)\right) .
$$

By using the independence of principal factors $W^{i}$, we have, for $t \in[0, T]$

$$
\begin{aligned}
\ln \left(\mathbb{E}\left(e^{s\left(d_{1} W_{t}^{1}+d_{2} W_{t}^{2}+\cdots+d_{m} W_{t}^{m}\right)}\right)\right) & =\ln \left(\prod_{j=1}^{m} \mathbb{E}\left(e^{s d_{j} W_{t}^{j}}\right)\right) \\
\sum_{j=1}^{m} \ln \left(\mathbb{E}\left(e^{s d_{j} W_{t}^{j}}\right)\right) & =t \sum_{j=1}^{m} \phi_{j}\left(s d_{j}\right),
\end{aligned}
$$

where the last equality comes from $\mathbb{E}\left(e^{s W_{t}^{j}}\right)=e^{t \phi_{j}(s)}$.

If we specialize the above equations to the case of EVaR, then Equation (28) becomes, for $t \in[0, T]$

$$
f_{E V a R_{\beta}}^{t}\left(u_{1}, u_{2}, \ldots, u_{n}\right)=\operatorname{EVaR}_{\beta}\left(-\left(d_{1} W_{t}^{1}+d_{2} W_{t}^{2}+\cdots+d_{m} W_{t}^{m}\right)\right)=\inf _{s \geq 0} \frac{t \sum_{j=1}^{m} \phi_{j}\left(s d_{j}\right)-\ln \beta}{s} .
$$

Now, consider the right-hand side of Equation (29). By taking derivatives with respect to $s$ we have, for $t \in[0, T]$,

$$
\frac{\partial}{\partial s}\left(\frac{t \sum_{j=1}^{m} \phi_{j}\left(s d_{j}\right)-\ln \beta}{s}\right)=\frac{s t \sum_{j=1}^{m} d_{j} \phi_{j}^{\prime}\left(s d_{j}\right)-t \sum_{j=1}^{m} \phi_{j}\left(s d_{j}\right)+\ln \beta}{s^{2}} .
$$

By setting Equation (30) equal to zero, we can find the value $s^{*}\left(t, u^{1}, \ldots, u^{n}\right)$ that minimizes the right-hand side in (29). As indicated by the notation, this minimum value $s^{*}\left(t, u^{1}, \ldots, u^{n}\right)$ is a 
function of $t$ and $u_{i}$ for $1 \leq i \leq n$ but in the following we use the more simple notation $s^{*}$ for this value. Notice that the value $s^{*}$ is in fact the infimum too. Based on convexity property of Laplace transform for one-sided Lévy processes and the condition $\phi_{j}^{\prime}(0) \geq 0$, the infimum in (29) should be reached at some point we denote $s^{*}$ (see [42]).

According to Theorem 5, the Euler allocation is the only possible allocation method for EVaR ${ }_{\beta}$. So, in order to find the capital allocation, it is sufficient to find the derivative of the right-hand side of Equation (29) with respect to the variable $u_{i}$ and evaluate it at the point $u=(1,1, \ldots, 1)$. Straight-forward differentiation yields, for $i=1, \ldots, n$ and $t \in[0, T]$

$$
\frac{\partial}{\partial u_{i}} f_{E V a R_{\beta}}^{t}\left(u_{1}, u_{2}, \ldots, u_{n}\right)=\frac{s^{*} t \sum_{j=1}^{m}\left(s_{i}^{*} d_{j}+a_{i j} s^{*}\right) \phi_{j}^{\prime}\left(s^{*} d_{j}\right)-t s_{i}^{*} \sum_{j=1}^{m} \phi_{j}\left(s^{*} d_{j}\right)+s_{i}^{*} \ln \beta}{s^{* 2}},
$$

where we use the notation $s_{i}^{*}=\frac{\partial s^{*}}{\partial u_{i}}$.

Since $s^{*}$ is the solution of setting Equation (30) equal to zero, we can simplify (31) as follows, for $i=1, \ldots, n$.

$$
\frac{\partial}{\partial u_{i}} f_{E V a R_{\beta}}^{t}\left(u_{1}, u_{2}, \ldots, u_{n}\right)=t \sum_{j=1}^{m} a_{i j} \phi_{j}^{\prime}\left(s^{*} d_{j}\right) \text {. }
$$

Evaluating Equation (32) at the point $u=(1,1, \ldots, 1)$ yields the allocated capital associated to the $i^{t h}$ department at time $t \in[0, T]$. Namely, for $i=1, \ldots, n$

$$
K_{t}^{i}=\frac{\partial}{\partial u_{i}} f_{E V a R_{\beta}}^{t}\left(u_{1}, u_{2}, \ldots, u_{n}\right)=t \sum_{j=1}^{m} a_{i j} \phi_{j}^{\prime}\left(s^{*} \sum_{k=1}^{n} a_{k j}\right) \text {. }
$$

Using Corollary 3 and integrating $K_{t}^{i}$ in (33) yields the allocated capital satisfying Definition 7 with respect to the risk measure $\mathrm{CEVa} R_{\beta}$. Thus, the allocated capital to $i^{\text {th }}$ department over the period $[0, T]$ with respect to $\mathrm{CEVaR}_{\beta}$ is

$$
L^{i}=\frac{1}{T} \int_{0}^{T} K_{t}^{i} d t-c^{i} \frac{T}{2}
$$

This completes the proof.

\section{Examples}

In this section, we are interested in examining the particular results and framework discussed in the previous section, in particular Theorem 6. Here, we do this for couple of examples in order to illustrate how this capital allocation can be computed.

As we will see, there are some particular models for which we can obtain an explicit expression for the capital allocation. In others, such an explicit form is not available but a solution can still be obtained by standard numerical methods. The difficulty lies in solving Equation (30) when is set to be equal to zero. We now discuss some particular models that have appeared in the literature on insurance Lévy risk processes and give explicit and semi-explicit expressions for their capital allocation.

\subsection{Brownian Motion with Scale Parameter}

Consider the general set-up defined through Equation (24). Let the principal factors $W_{t}^{1}, \ldots, W_{t}^{m}$ to be $m$ independent Brownian motions with different scale parameters $\sigma_{i}>0$ and m.g.f $\mathbb{E}\left(e^{s W_{t}^{i}}\right)=e^{\frac{1}{2} \sigma_{i}^{2} s^{2} t}$. We now only need to apply Theorem 6. By solving Equation (30) equal to zero we get, for $t \in[0, T]$

$$
s^{2} t \sum_{j=1}^{m} d_{j}^{2} \sigma_{j}^{2}-\frac{1}{2} s^{2} t \sum_{j=1}^{m} d_{j}^{2} \sigma_{j}^{2}+\ln \beta=0,
$$


where $d_{j}$ is given in (27). Or equivalently,

$$
s^{*}=\left(\frac{-2 \ln \beta}{t \sum_{j=1}^{m} d_{j}^{2} \sigma_{j}^{2}}\right)^{\frac{1}{2}} .
$$

Substituting (35) into Equation (33) at the point $u=(1,1, \ldots, 1)$ we can compute the value $K_{t}^{i}$ for $i=1, \ldots, n$. That is,

$$
K_{t}^{i}=t^{\frac{1}{2}}\left(\frac{-2 \ln \beta}{\sum_{j=1}^{m} \sigma_{j}^{2}\left(\sum_{k=1}^{n} a_{k j}\right)^{2}}\right)^{\frac{1}{2}} \sum_{j=1}^{m} \sigma_{j}^{2} a_{i j} \sum_{k=1}^{n} a_{k j},
$$

for $t \in[0, T]$. Thus, the allocated capital to the $i^{\text {th }}$ department with respect to $\mathrm{CEVaR}_{\beta}$ can be computed to be

$$
L^{i}=\frac{2}{3} T^{\frac{1}{2}}\left(\frac{-2 \ln \beta}{\sum_{j=1}^{m} \sigma_{j}^{2}\left(\sum_{k=1}^{n} a_{k j}\right)^{2}}\right)^{\frac{1}{2}} \sum_{j=1}^{m} \sigma_{j}^{2} a_{i j} \sum_{k=1}^{n} a_{k j}-c^{i} \frac{T}{2} .
$$

Now as a special case, let the principal factors $W_{t}^{1}, \ldots, W_{t}^{m}$ to be $m$ independent Brownian motions with common scale parameter $\sigma>0$ and common Laplace transform $\mathbb{E}\left(e^{s W_{t}^{i}}\right)=e^{\frac{1}{2} \sigma^{2} s^{2} t}$. So, (35) will be reduced to

$$
s^{*}=\left(\frac{-2 \ln \beta}{\sigma^{2} t \sum_{j=1}^{m} d_{j}^{2}}\right)^{\frac{1}{2}},
$$

and the value $K_{t}^{i}$ is then, for $t \in[0, T]$

$$
K_{t}^{i}=\sigma t^{\frac{1}{2}}\left(\frac{-2 \ln \beta}{\sum_{j=1}^{m}\left(\sum_{k=1}^{n} a_{k j}\right)^{2}}\right)^{\frac{1}{2}} \sum_{j=1}^{m} a_{i j} \sum_{k=1}^{n} a_{k j} .
$$

Thus, the allocated capital, $L^{i}$, to the $i^{\text {th }}$ department satisfying Definition 7 with respect to $\mathrm{CEVaR}_{\beta}$ for this special case can be written as

$$
L^{i}=\frac{2}{3} T^{\frac{1}{2}} \sigma\left(\frac{-2 \ln \beta}{\sum_{j=1}^{m}\left(\sum_{k=1}^{n} a_{k j}\right)^{2}}\right)^{\frac{1}{2}} \sum_{j=1}^{m} a_{i j} \sum_{k=1}^{n} a_{k j}-c^{i} \frac{T}{2} .
$$

\subsection{Cramér-Lundberg Process}

Consider again the general set-up defined through Equation (24). We let the principal factors $W_{t}^{1}, \ldots, W_{t}^{m}$ to be $m$ independent compound Poisson processes with different jump means $\frac{1}{\mu_{i}}>0$. i.e.,

$$
W_{t}^{i}=\sum_{k=1}^{N_{t}^{i}} Z_{k}^{i}
$$

where the number of claims is assumed to follow a Poisson process $\left(N_{t}^{i}\right)_{0 \leq t \leq T}$ with intensity $\lambda_{i}$ which is independent of the positive and i.i.d random variables $\left(Z_{n}^{i}\right)_{n \geq 1}$ representing claim sizes. The m.g.f for (41) is

$$
\mathbb{E}\left(e^{s W_{t}^{i}}\right)=\exp \left[\lambda_{i}\left(\psi_{i}(s)-1\right)\right],
$$


where $\psi_{i}(s)$ is the m.g.f for claim process for $i=1, \ldots, m$. By solving Equation (30) equal to zero we get, for $t \in[0, T]$

$$
t \sum_{j=1}^{m} \lambda_{j}\left(s d_{j} \psi_{j}^{\prime}\left(s d_{j}\right)-\psi_{j}\left(s d_{j}\right)+1\right)+\ln \beta=0,
$$

where $d_{j}$ is given in (27). Let $s^{*}$ be a solution of (43) satisfying (29) which also is a function of $t$. Evaluating $s^{*}$ at the point $u=(1,1, \ldots, 1)$ and substituting into (33) yields the capital allocation value $K_{t}^{i}$ for $i=1, \ldots, n$. That is,

$$
K_{t}^{i}=t \sum_{j=1}^{m} a_{i j} \lambda_{j} \psi_{j}^{\prime}\left(s^{*} \sum_{k=1}^{n} a_{k j}\right)
$$

for $t \in[0, T]$. Thus, the allocated capital to the $i^{\text {th }}$ department satisfying Definition 7 with respect to $\mathrm{CEVaR}_{\beta}$ is given by

$$
L^{i}=\frac{1}{T} \int_{0}^{T} K_{t}^{i} d t-c^{i} \frac{T}{2}
$$

for $i=1, \ldots, n$. As a special case assume that claim sizes follow exponential distributions with mean $\frac{1}{\mu_{i}}$ for $i=1, \ldots, m$. In this case we have $\psi^{i}(s)=\frac{\mu_{i}}{\mu_{i}-s}$ for $s<\mu_{i}$. Therefore, Equation (44) will be reduced to the following equation for $t \in[0, T]$.

$$
s t \sum_{j=1}^{m} d_{j} \lambda_{j}\left(\frac{\mu_{j}}{\left(\mu_{j}-s d j\right)^{2}}-\frac{1}{\mu_{j}-s d j}\right)+\ln \beta=0, \quad s<\min \left(\frac{\mu_{j}}{d_{j}}\right)_{1 \leq j \leq m},
$$

where $d_{j}$ is given in (27). This is not as straight-forward as the equivalent equation for the previous example. Nonetheless, the value $s^{*}$ satisfying (46) and (29) can be obtained numerically. Evaluating at the point $u=(1,1, \ldots, 1)$ and substituting into (33) yields the capital allocation value $K_{t}^{i}$ for $i=1, \ldots, n$. That is,

$$
K_{t}^{i}=t \sum_{j=1}^{m} a_{i j}\left(\frac{\lambda_{j} \mu_{j}}{\left(\mu_{j}-s^{*} \sum_{k=1}^{n} a_{k j}\right)^{2}}\right),
$$

for $t \in[0, T]$ and where $s^{*}$ is the solution of Equation (46). Thus, the allocated capital to the $i^{\text {th }}$ department satisfying Definition 7 with respect to $C E V a R_{\beta}$ is given by

$$
L^{i}=\frac{1}{T} \int_{0}^{T} K_{t}^{i} d t-c^{i} \frac{T}{2}
$$

for $1 \leq i \leq n$.

\subsection{Gamma Subordinator}

Assume the general set-up defined through Equation (24). We let the principal factors $W_{t}^{1}, \ldots, W_{t}^{m}$ to be $m$ independent gamma processes with different parameters $\alpha_{i}, b_{i}>0$ and m.g.f

$$
\mathbb{E}\left(e^{s W_{t}^{i}}\right)=\left(1-\frac{s}{b_{i}}\right)^{-\alpha_{i} t}=\exp \left[-t \alpha_{i} \ln \left(1-\frac{s}{b_{i}}\right)\right], \quad s<b_{i} .
$$

We now only need to apply Theorem 6. By solving Equation (30) equal to zero we get, for $t \in[0, T]$

$$
t \sum_{j=1}^{m} \alpha_{j}\left(\ln \left(1-\frac{s d_{j}}{b_{j}}\right)+s \frac{d_{j}}{b_{j}-s d_{j}}\right)+\ln \beta=0, \quad s<\min \left(\frac{b_{j}}{d_{j}}\right)_{1 \leq j \leq m},
$$

where $d_{j}$ is given in (27). This equation like Equation (46) is not as straight-forward as the equivalent equation for the example with Brownian motion. Nonetheless, the value $s^{*}$ satisfying (50) and (29) 
can be obtained numerically. Evaluating at the point $u=(1,1, \ldots, 1)$ and substituting into (33) yields the capital allocation value $K_{t}^{i}$ for $i=1, \ldots, n$. That is,

$$
K_{t}^{i}=t \sum_{j=1}^{m} a_{i j}\left(\frac{\alpha_{j}}{b_{j}-s^{*} \sum_{k=1}^{n} a_{k j}}\right)
$$

for $t \in[0, T]$ and where $s^{*}$ is the solution of Equation (50). Thus, the allocated capital to the $i^{t h}$ department satisfying Definition 7 with respect to $C E V a R_{\beta}$ is given by

$$
L^{i}=\frac{1}{T} \int_{0}^{T} K_{t}^{i} d t-c^{i} \frac{T}{2}
$$

for $1 \leq i \leq n$.

\section{Conclusions}

The contribution of this paper is two-fold. With a view towards capital allocation applications, we first introduce a new coherent cumulative risk measure on a subclass in the space of càdlàg processes that has the feature of being tractable enough within a class of models, namely spectrally positive Lévy insurance risk processes. This first contribution is significant since it circumvents the technical difficulties associated with the problem of capital allocation for risk measures on the space of risk processes. By introducing an ad-hoc risk measure, we are able to give closed-form expressions for the capital allocation problem in this framework. Our results are discussed within the current approaches and connections to existing alternatives can be pointed out. A second contribution is the illustration of the tractability of our results on the capital allocation problem for stochastic processes in an insurance context. We showed that the only method to allocate the requiring capital for this risk measure is a tractable extension of the Euler allocation method. Moreover, we provide illustrations of our results in an insurance context for a model inspired from the CreditRisk+ model. Indeed, we find explicit and semi-explicit expressions for the capital allocation under well-known models for the aggregate claims processes such as Brownian motion and Cramer-Lundberg model. This contribution focused on the first technical and crucial step of the proposed methodology that allows to derive tractable expressions for the capital allocation. Future work is needed on the practical and numerical implementation of such models and expressions. We believe, the flexibility in definition $\mathrm{CEVAR}_{\beta}$ proposed in Equation (11) paves the ground to study this risk measure in other contexts as well such as in finance. Extensions can also be studied, for instance one can propose an appropriate weight function and replace it in Equation (11) to come up with another risk measure.

Author Contributions: All three authors collaborated equally at every step of the research and in writing the paper.

Conflicts of Interest: The authors declare no conflict of interest.

\section{References}

1. Cramér, H. Historical review of Filip Lundberg's works on risk theory. Scand. Actuar. J. 1969, 52, 6-9.

2. Asmussen, S. Ruin Probabilities; Volume 2 of Advanced Series on Statistical Science \& Applied Probability; World Scientific Publishing Co. Inc.: River Edge, NJ, USA, 2000.

3. Biffis, E.; Morales, M. On a generalization of the Gerber-Shiu function to path-dependent penalties. Insur. Math. Econ. 2009, 46, 92-97.

4. Biffis, E.; Kyprianou, A. A Note on Scale Functions and the Time Value of Ruin for Lévy Insurance Risk Processes. Insur. Math. Econ. 2010, 46, 85-91.

5. Trufin, J.; Albrecher, H.; Denuit, M. Properties of a Risk Measure Derived from Ruin Theory. Geneva Risk Insur. Rev. Palgrave 2011, 36, 174-188.

6. Loisel, S.; Trufin, J. Properties of a risk measure derived from the expected area in red. Insur. Math. Econ. 2014, 55, 191-199. 
7. Dhaene, J.; Tsanakas, A.; Valdez, E.A.; Vanduffel, S. Optimal capital allocation principles. J. Risk Insur. 2012, $79,1-28$.

8. Cheridito, P.; Delbaen, F.; Kupper, M. Coherent and convex monetary risk measures for bounded càdlàg processes. Stoch. Process. Appl. 2004, 112,1-22.

9. Cheridito, P.; Delbaen, F.; Kupper, M. Coherent and convex monetary risk measures for unbounded càdlàg processes. Financ. Stoch. 2005, 9, 369-387.

10. Cheridito, P.; Delbaen, F.; Kupper, M. Dynamic monetary risk measures for bounded discrete-time processes. Electron. J. Probab. 2006, 11, 57-106.

11. Cummins, J.D. Allocation of capital in the insurance industry. Risk Manag. Insur. Rev. 2000, 3, 7-27.

12. Venter, G.G. Capital allocation survey with commentary. N. Am. Actuar. J. 2004, 8, 96-107.

13. Urban, M.; Dittrich, J.; Kluppelberg, C.; Stolting, R. Allocation of Risk Capital to Insurance Portfolios; Blatter der DGVFM; Springer-Berlin: Heidelberg, Germany, 2004; pp. 389-406.

14. Valdez, E.A. Principles and methods of capital allocation for enterprise risk management. In Proceedings of the Spring School on Risk Management, Insurance and Finance European University, St. Petersburg, Russia, 2-4 April 2012.

15. LeMaire, J. An application of game theory: Cost allocation. ASTIN Bull. 1984, 14, 61-81.

16. Denault, M. Coherent allocation of risk capital. J. Risk 2001, 4, 1-34.

17. Tasche, D. Risk Contributions and Performance Measurement; Technical document; Munich University of Technology: Munich, Germany, 1999.

18. Panjer, H.H. Measurement of Risk, Solvency Requirements, and Allocation of Capital Within Financial Conglomerates; Technical report, Research Report 01-14; Institute of Insurance and Pension Research, University of Waterloo: Waterloo, ON, Canada, 2001.

19. Landsman, Z.; Valdez, E.A. Tail conditional expectations for elliptical distribution. N. Am. Actuar. J. 2003, 7, 55-71.

20. Tsanakas, A. Dynamic capital allocation with distortion risk measures. Insur. Math. Econ. 2004, 35, $223-243$.

21. Dhaene, J.; Henrard, L.; Landsman, Z.; Vandendorpe, A.; Vanduffel, S. Some results on the CTE-based capital allocation rule. Insur. Math. Econ. 2008, 42, 855-863.

22. Kim, J.H.T.; Hardy, M.R. A capital allocation based on a solvency exchange option. Insur. Math. Econ. 2009, 44, 357-366.

23. Tsanakas, A. To split or not to split: Capital allocation with convex risk measures. Insur. Math. Econ. 2009, 44, 268-277.

24. Furman, E.; Zitikis, R. Weighted risk capital allocations. Insur. Math. Econ. 2008, 43, 263-269.

25. Kalkbrener, M. An axiomatic characterization of capital allocations of coherent risk measures. Quant. Financ. 2009, 9, 961-965.

26. Biard, R. Asymptotic multivariate finite-time ruin probabilities with heavy-tailed claim amounts: Impact of dependence and optimal reserve allocation. Bull. Francais d'Actuariat, Institut des Actuaires 2013, 13, 79-92.

27. Tsanakas, A.; Millossovich, P. Sensitivity Analysis Using Risk Measures. Risk Anal. 2016, 36, 30-48.

28. Mizgier, K.J.; Pasia, J.M. Multi objective optimization of credit capital allocation in financial institutions. Centr. Eur. J. Oper. Res. 2015, in press.

29. Erel, I.; Myers, S.C.; Read, J.A. A theory of risk capital. J. Financ. Econ. 2015, 118, 620-635.

30. Assa, H. Lebesgue property of risk measures for bounded cádlág processes and applications. Methods Appl. Anal. 2012, 18, 335-350.

31. Ahmadi-Javid, A. Entropic Value-at-Risk: A New Coherent Risk Measure. J. Optim. Theory Appl. 2012, 155, 1105-1123.

32. Assa, H. On Some Aspects of Coherent Risk Measures and their Applications. Ph.D. Thesis, Université de Montréal, Montréal, QC, Canada, 4 August 2011.

33. Follmer, H.; Knispel, T. Entropic risk measures: Coherence vs. convexity, model ambiguity, and robust large deviations. Stoch. Dyn. 2011, 11, 333-351.

34. Sato, K.-I. Lévy Processes and Infinitely Divisible Distributions; Cambridge University Press: Cambridge, UK, 1999.

35. Follmer, H.; Schied, A. Stochastic Finance: An Introduction in Discrete Time; Volume 27 of de Gruyter Studies in Mathematics, extended ed.; Walter de Gruyter \& Co.: Berlin, Germany, 2004.

36. Cherny, A. Pricing with coherent risk. Transl. Theory Probab. Appl. 2008, 52, 389-415. 
37. Delbaen, F. Coherent Monetary Utility Functions. Available online: https://people.math.ethz.ch/delbaen/ ftp/preprints/PISA007.pdf (accessed on 10 August 2016).

38. Fischer, T. Risk Capital Allocation by Coherent Risk Measures Based on One-Sided Moments. Insur. Math . Econ. 2003, 32, 135-146.

39. Billera, L.J.; Heath, D.C. Allocation of shared costs: A set of axioms yielding a unique procedure. Math. Oper. Res. 1982, 7, 32-39.

40. Rockafellar, R. Convex Analysis; Princeton University Press: Princeton, NJ, USA, 1970.

41. Gundlach, M.; Lehrbass, F. (Eds.) CreditRisk+ in the Banking Industry; Springer: Berlin, Germany, 2004.

42. Kyprianou, A.E. Introductory Lecture Notes on Fluctuations of Lévy processes with Applications; Springer-Verlag: Berlin/Heidelberg, Germany, 2006.

(C) 2016 by the authors; licensee MDPI, Basel, Switzerland. This article is an open access article distributed under the terms and conditions of the Creative Commons Attribution (CC-BY) license (http://creativecommons.org/licenses/by/4.0/). 